\title{
Observation of $\mathrm{SiO}_{2}$ Nanoparticle Formation via UV Pulsed Laser
}

\author{
Ablation in a Background Gas \\ Reiji Koike $^{1}$, Rio Suzuki ${ }^{1}$, Keita Katayama ${ }^{1}$, Mitsuhiro Higashihata ${ }^{1}$, Hiroshi Ikenoue ${ }^{1,2}$, and Daisuke Nakamura ${ }^{* 1}$ \\ ${ }^{1}$ Graduate School of Information Science and Electrical Engineering, Kyushu University, Japan \\ ${ }^{2}$ Department of Gigaphoton Next GLP, Kyushu University, Japan \\ *Corresponding author's e-mail: dnakamura@ees.kyushu-u.ac.jp
}

\begin{abstract}
$\mathrm{SiO}_{2}$ nanoporous films have recently gained significant popularity as low-k dielectric constant insulating films. In this study, we demonstrated the fabrication of a low- $\mathrm{k} \mathrm{SiO}_{2}$ nanoporous film consisting of $\mathrm{SiO}_{2}$ nanoparticles by pulsed laser deposition. However, to optimize the growth conditions of nanoporous films, it is important to understand the spatial and size distribution of nanoparticles produced by laser ablation. Here, we visualized laser-ablated $\mathrm{SiO}_{2}$ nanoparticles using a two-dimensional laser scattering imaging technique and investigated the influence of gas species and pressure on the spatial distribution of the nanoparticles in the gas phase. After the decay of the plasma emission within several microseconds, a spherical-shaped scattering image depicting the spatial distribution of $\mathrm{SiO}_{2}$ nanoparticles was obtained from a delay time of approximately $10 \mathrm{~ms}$. In Ar gas, the sphericalshaped distribution of $\mathrm{SiO}_{2}$ nanoparticles changed to an ellipsoidal shape with increasing pressure, whereas in $\mathrm{O}_{2}$ gas, it changed a mushroom-like shape. These results show that the gas species and pressure are dominant parameters affecting the spatial distribution of nanoparticle formation.
\end{abstract}

DOI: $10.2961 / \mathrm{j} 1 \mathrm{mn} .2021 .03 .2006$

Keywords: nanoparticles, laser ablation, nanoporous film.

\section{Introduction}

Over the past four decades, ultra-large-scale integrated circuits (ULSIs) have become more compact in accordance with Moore's law [1-3], and the gate delay time and power consumption have decreased. However, the $R C$ delay time increases owing to wiring, which increases the total delay time [4]. One way to solve this problem is to reduce the capacitance between the interconnections, which requires a low-k material as an interlayer dielectric. In addition, low-k interlayer dielectrics are required to have various material properties such as heat and moisture resistance, and mechanical strength. To fabricate low-k interlayer dielectrics that satisfy the required properties, various materials such as $\mathrm{SiO}_{2}$-based $\mathrm{SiOC}[5]$ and $\mathrm{SiOCH}[6]$ have been developed. In addition, many interlayer dielectric formation and planarization techniques have been studied, such as adopting porous films itself porous, or inserting an air gap [7].

We focused on low-k porous films manufactured by depositing nanoparticles using pulsed laser deposition (PLD). PLD is widely used as a method to fabricate thin films. In recent years, it has been used to produce thin-film oxides [8] and compounds $[9,10]$. In addition, PLD can produce nanoparticles, as well as nanoporous films consisting of nanoparticles [11]. In PLD, the characteristics of the fabricated thin film are generally strongly affected by the growth conditions, such as gas pressure, gas species, and the distance between the target and substrate. In the case of nanoparticle deposition using PLD, nanoparticle formation in the gas phase is dramatically affected by the growth conditions. Therefore, understanding the spatial and size distribution of nanoparticles produced by laser ablation is important for establishing the preferred growth conditions for nanoporous films. Particularly, gas species and gas pressure strongly affect the spatial distribution of nanoparticles formed by the laser ablation [12]. The purpose of this study is to investigate the spatial distribution of nanoparticles under different gas species and pressures using a two-dimensional laser scattering imaging technique [12-16].

\section{EXPERIMENT}

The experimental setup for the time-resolved measurement of $\mathrm{SiO}_{2}$ nanoparticles is shown in Fig. 1 [17]. $\mathrm{A} \mathrm{SiO}_{2}$ target on a rotating holder in a vacuum chamber filled with $\mathrm{Ar}$ or $\mathrm{O}_{2}$ gas was ablated by an $\mathrm{ArF}$ excimer laser

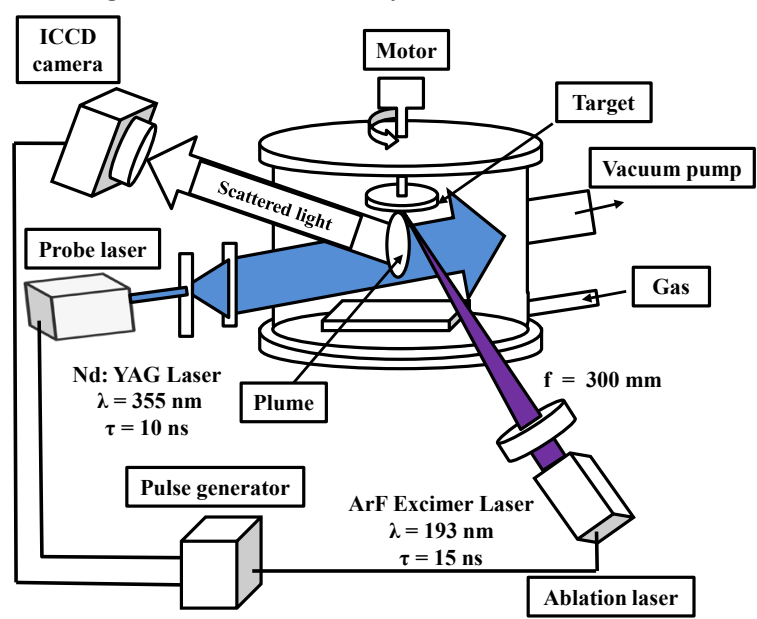

Fig.1 Experimental setup of the 2-D laser imaging technique. 
( $\lambda=193 \mathrm{~nm}$, pulse width $\tau=15 \mathrm{~ns}$, COMPex PRO $102 \mathrm{~F}$ ). The laser beam was extracted through a $5 \mathrm{~mm}$ diameter aperture and focused by a lens onto the target placed at the top of the vacuum chamber. The ablation fluence was $6 \mathrm{~J} / \mathrm{cm}^{2}$ $\left(5.2 \mathrm{~mJ}\right.$ on $\left.0.087 \mathrm{~mm}^{2}\right)$, and the laser was operated with a single pulse. The gas was regulated between 400-2700 $\mathrm{Pa}$ and refreshed after each shot to prevent disturbance from particles produced by the former laser pulse. A Nd: YAG laser $(\lambda=355 \mathrm{~nm}$, pulse width $\tau=10 \mathrm{~ns}$, Quanta-ray GCR200 , Spectra-Physics) was used as the probe laser to observe Rayleigh scattering of nanoparticles produced by the ablation laser. The probe beam was shaped into a sheet with a width of approximately $30 \mathrm{~mm}$ using cylindrical lenses, which passed parallel to the target surface at approximately $2 \mathrm{~mm}$ from the target. The polarization direction of the probe laser was set parallel to the observation plane. The fluence was $0.26 \mathrm{~J} / \mathrm{cm}^{2}\left(32 \mathrm{~mJ}\right.$ on $\left.12.3 \mathrm{~mm}^{2}\right)$ and the laser was again operated with a single pulse. The scattered light from the $\mathrm{SiO}_{2}$ nanoparticles was observed using an intensified charge-coupled device (ICCD, Roper PI-MAX) camera. The ablation laser, probe laser, and ICCD camera were connected to a pulse generator to adjust for the delays in each device. By changing the delay time of the probe lasers of the ablation laser, the temporal variation of the spatial distribution of $\mathrm{SiO}_{2}$ nanoparticles was obtained.

\section{Results and discussion}

Before observing the scattering light from the laser-produced particles, the plasma emission produced by the ablation laser was measured using an ICCD camera without any probe laser irradiation. Fig. 2 shows the plasma emission images in $\mathrm{Ar}$ at $400 \mathrm{~Pa}$. The white dotted line indicates the location of the target holder. The colored bar indicates the signal intensity, and the delay time is shown on the lower left

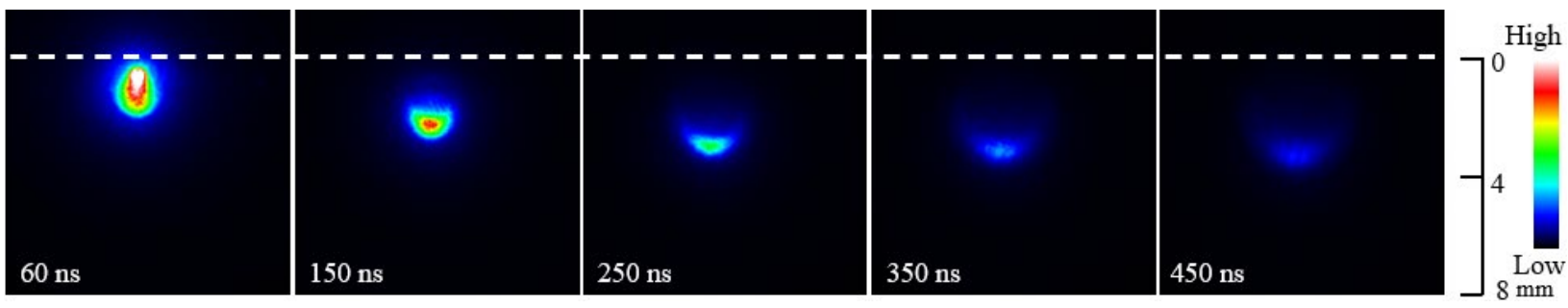

Fig. 2 Plasma emission image in Ar gas at $400 \mathrm{~Pa}$.

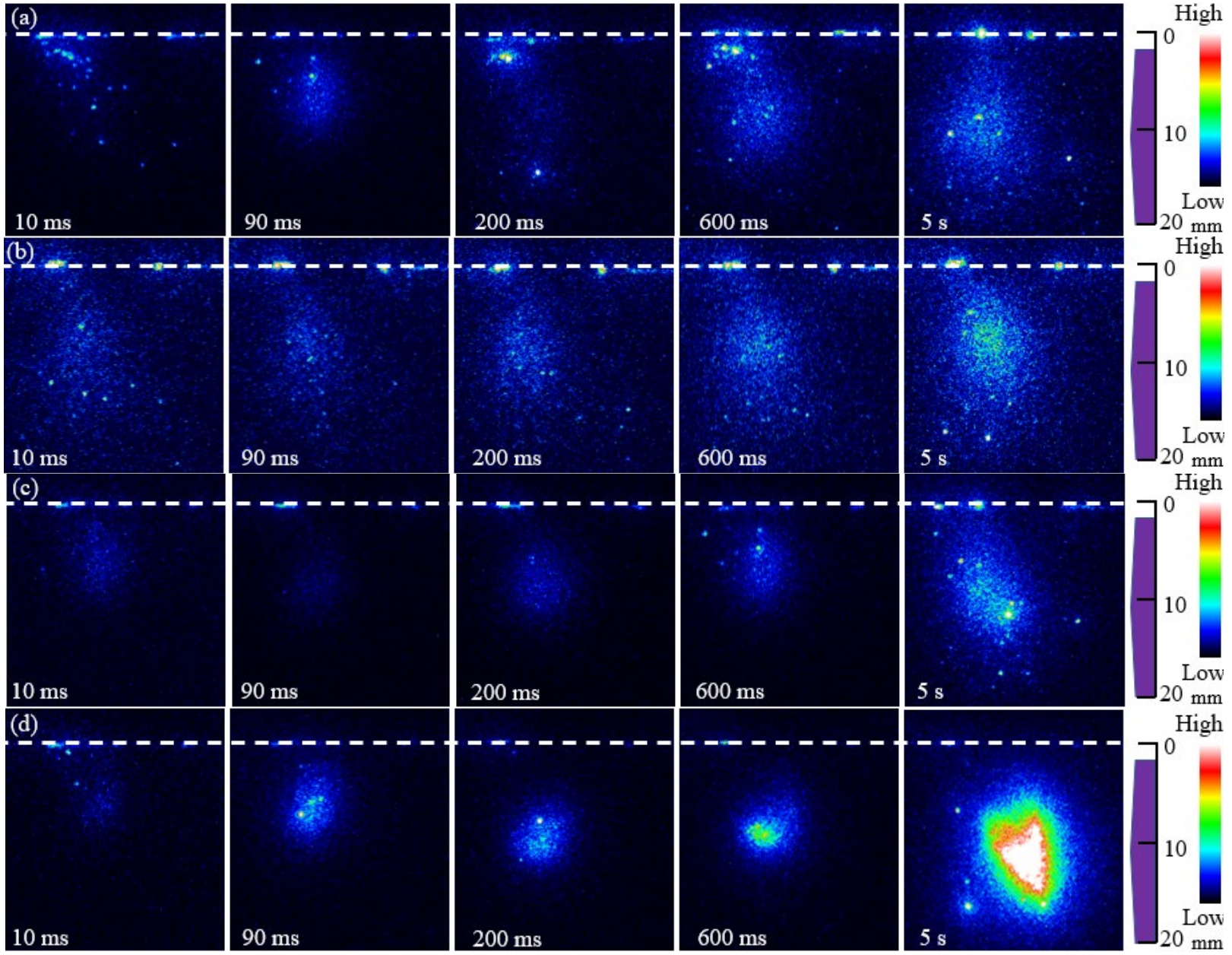

Fig. 3 Temporal changes of spatial distribution of $\mathrm{SiO}_{2}$ nanoparticles in Ar gas at (a) $400 \mathrm{~Pa}$, (b) $670 \mathrm{~Pa}$, (c) $1300 \mathrm{~Pa}$ and (d) $2700 \mathrm{~Pa}$. 
part of each image. The plasma emission disappeared at approximately $5 \mu \mathrm{s}$ in Ar at $400 \mathrm{~Pa}$. The emission duration was extended by increasing the gas pressure to $670 \mathrm{~Pa}$ at $6 \mu \mathrm{s}$, $1300 \mathrm{~Pa}$ at $8 \mu \mathrm{s}$, and $2700 \mathrm{~Pa}$ at $10 \mu \mathrm{s}$. Regarding $\mathrm{O}_{2}$ gas, similar images were observed to those observed in Ar gas. However, the emission duration was shorter than that observed in Ar gas, namely approximately $2 \mu \mathrm{s}$ at $400 \mathrm{~Pa}, 3 \mu \mathrm{s}$ at $670 \mathrm{~Pa}$ and $1300 \mathrm{~Pa}$, and $4 \mu \mathrm{s}$ at $2700 \mathrm{~Pa}$. This result suggests that the plasma emission is dependent on the gas species and gas pressure, and the specific heat of the background gas may influence the cooling process of the plume. In addition, while the time range in which the plasma emission was observed, no scattering light was observed with the probe laser irradiation, indicating that very few nanoparticles were formed in the microsecond range after laser ablation.

Furthermore, scattering light from the particles with a delay time in the millisecond range was observed. The scattering phenomena originate from nanoparticles formed by aggregation of the laser-ablated species in the plume owing to collision with gas molecules and with each other. Because the photon energy of the ablation laser is lower than the bandgap energy of $\mathrm{SiO}_{2}$, the incident laser light is partially transparent to the target. Thus, the stability of each ablation phenomenon is poor. In the experiment, the reproducibility of the ablation phenomenon was improved by irradiating the laser multiple times to the same spot. The spatial distribution images of $\mathrm{SiO}_{2}$ nanoparticles under different $\mathrm{Ar}$ gas pressures, namely, $400 \mathrm{~Pa}, 670 \mathrm{~Pa}, 1300 \mathrm{~Pa}$, and $2700 \mathrm{~Pa}$ are shown in Fig. 3. The scales on the right-hand side of the images indicate the distance from the target, and the colored areas indicate the region illuminated by the sheet probe laser beam. In Ar gas, scattered light was observed from $10 \mathrm{~ms}$ at $400 \mathrm{~Pa}, 670 \mathrm{~Pa}, 1300 \mathrm{~Pa}$ and $2700 \mathrm{~Pa}$. Subsequently, the scattered light intensity increased as the delay time elapsed. This is because the signal intensity of Rayleigh scattering is proportional to $d^{6} N$, where $d$ is the diameter of the particle and $N$ is the number density of the particle. In the literature [18], nanoparticle formation owing to collisions between the laser-ablated species and background gas molecules is in the order of microseconds, and the size of nanoparticles is assumed to grow on this time scale. Because the time scale of the images shown in Fig. 3 is in the order of milliseconds, the increase in the number density of nanoparticles is considered dominant. The spatial distributions of the nanoparticles at $400 \mathrm{~Pa}, 670 \mathrm{~Pa}$, and $1300 \mathrm{~Pa}$ were spherical. At a higher pressure, $2700 \mathrm{~Pa}$, the distribution was ellipsoidal. Surprisingly, the nanoparticles maintained their spatial

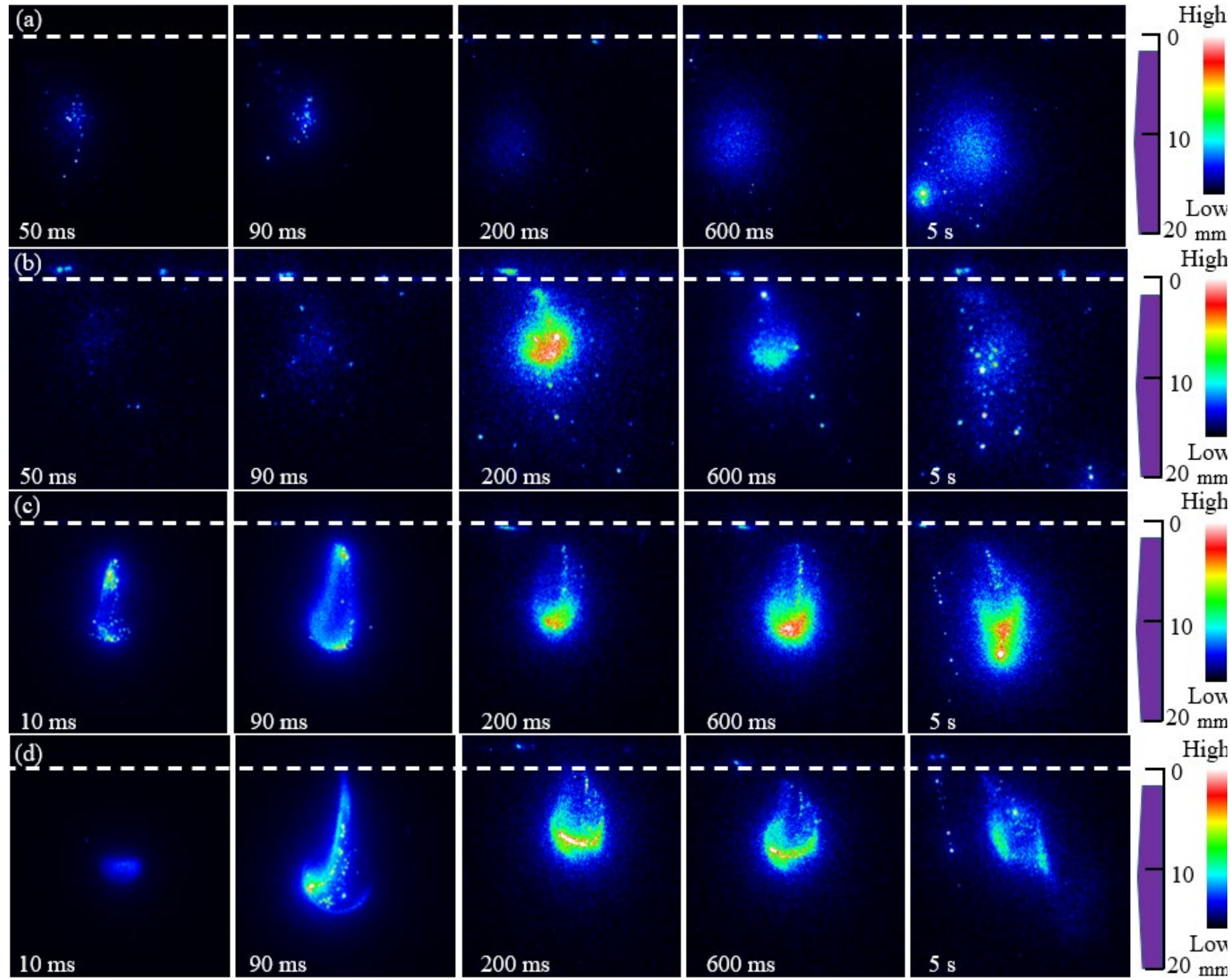

Fig. 4 Temporal changes of spatial distribution of $\mathrm{SiO}_{2}$ nanoparticles in $\mathrm{O}_{2}$ gas at (a) $400 \mathrm{~Pa}$, (b) $670 \mathrm{~Pa}$, (c) $1300 \mathrm{~Pa}$ and (d) $2700 \mathrm{~Pa}$. 
distribution with slight diffusion for more than a few seconds. In addition, we confirmed that the scattered light from the spatial distribution was polarized, which indicates that the observed phenomenon was Rayleigh scattering from the $\mathrm{SiO}_{2}$ nanoparticles.

The spatial distribution images of $\mathrm{SiO}_{2}$ nanoparticles in $\mathrm{O}_{2}$ gas at $400 \mathrm{~Pa}, 670 \mathrm{~Pa}, 1300 \mathrm{~Pa}$, and $2700 \mathrm{~Pa}$ are shown in Fig. 4. At $400 \mathrm{~Pa}$ and $670 \mathrm{~Pa}$, the scattered light from the nanoparticles were observed from $50 \mathrm{~ms}$ and $30 \mathrm{~ms}$, respectively, whereas at $1300 \mathrm{~Pa}$ and $2700 \mathrm{~Pa}$, it was observed from $10 \mathrm{~ms}$. This was due to the enhancement of nanoparticle aggregation with higher gas pressure. The spatial distribution of the nanoparticles was spherical at $400 \mathrm{~Pa}$ and 670 $\mathrm{Pa}$. In contrast, a mushroom-like distribution was observed at $1300 \mathrm{~Pa}$ and $2700 \mathrm{~Pa}$. The spatial distribution in $\mathrm{O}_{2}$ gas was maintained more than a few seconds. This result confirms that the gas pressure depends on the spatial distribution of the nanoparticles, even under $\mathrm{O}_{2}$ gas conditions. In addition, these images indicate that the ablated species lost their initial kinetic energy owing to collisions with gas molecules within microseconds.

Comparing the spatial distribution of nanoparticles in each gas, the distribution changes from spherical to ellipsoidal shape with increasing pressure in $\mathrm{Ar}$ gas, whereas it changes from spherical to mushroom-like shape in $\mathrm{O}_{2}$ gas. This indicates that the spatial distribution of the nanoparticles is dependent on the gas species. A previous report on $\mathrm{Si}$ target ablation [12] showed the various spatial distributions of Si nanoparticles in different background gases, demonstrating that the spatial distribution of nanoparticles is not always determined by the relative atomic or molecular weight between the background gas and ablated atoms. In addition, our observation results of $\mathrm{SiO}_{2}$ nanoparticle distribution in $\mathrm{Ar}$ or $\mathrm{O}_{2}$ gases were different from those of Si nanoparticle distribution [12], indicating that the target material may affect the spatial distribution formation. Another report documented that the viscosity of the background gas may be crucial in the plume formation dynamics [18].

Finally, we measured the particle size deposited on the substrate using transmittance electron microscopy (TEM). The particle sizes deposited at 2.8, 4.0, and $9.0 \mathrm{~mm}$ from the target surface in $\mathrm{Ar}$ or $\mathrm{O}_{2}$ gas at $400 \mathrm{~Pa}$ was 5-30 nm for all deposition distances. However, we could not confirm the dependence of gas species or distance on the particle size.

\section{Conclusion}

We investigated the effect of gas species and gas pressure on the spatial distribution of $\mathrm{SiO}_{2}$ nanoparticles produced by laser ablation in the gas phase. After the decay of the plasma emission within several microseconds, a scattering image indicating the spatial distribution of $\mathrm{SiO}_{2}$ nanoparticles was obtained from a delay time of approximately
$10 \mathrm{~ms}$. In Ar gas, the spatial distribution changed from spherical to ellipsoidal with increasing pressure, whereas it changed from a spherical shape to mushroom-like shape in $\mathrm{O}_{2}$ gas. These results suggest that the viscosity and specific heat ratio of the background gas may affect the spatial distribution of the $\mathrm{SiO}_{2}$ nanoparticles. From TEM observations, the size of the $\mathrm{SiO}_{2}$ nanoparticles produced in $\mathrm{Ar}$ or $\mathrm{O}_{2}$ gas at $400 \mathrm{~Pa}$ was 5-30 $\mathrm{nm}$. In future research, we will investigate the size and spatial distribution of nanoparticles at a lower gas pressure range of $0.1-10 \mathrm{~Pa}$.

\section{References}

[1] W. Shockley, M. Sparks, and G. K. Teal: Phys. Rev., 83, (1951) 151.

[2] J. S. Kilby: U. S. Patent 3138743 (1964).

[3] T. Mizuno, J. Okamura, and A. Toriumi: IEEE Trans. Electron Devices, 41, (1994) 2216.

[4] S. Muthukumar, C. D. Hill, S. Ford, W. Worwag, T. Dambrauskas, P. C. Challela, T. S. Dory, N. M. Patel, E. L. Ramsay, and D. S. Chau: Proc. Electron. Compon. Technol. Conf. (2006).

[5] C.-C. Chiang, M.-C. Chen, L.-J. Li, Z.-C. Wu, S.-M. Jang, and M.-S. Liang: J. Electrochem. Soc., 151, (2004) G612.

[6] K. Maex, M. R. Baklanov, D. Shamiryan, F. lacopi, S. H. Brongersma, and Z. S. Yanovitskaya: J. Appl. Phys., 93, (2003) 8793.

[7] J. Noguchi, T. Oshima, T. Matsumoto, S. Uno, and K. Sato: IEEE Trans. Electron Devices, 56, (2009) 2675.

[8] A. A. Menazea, and A. M. Mostafa: J. Environ. Chem. Eng., 8, (2020) 104104.

[9] A. A. Menazea, and A. M. Mostafa: Radiat. Phys. Chem., $176,(2020) 109020$.

[10] A. A. Menazea, and N. S. Awwad: Radiat. Phys. Chem., 177, (2020) 109112.

[11] S. Saha, S. K. Arya, S. P. Singh, K. Sreenivas, B. D. Malhotra, and V. Gupta: Biosensors and Bioelectronics, 24, (2009) 2040.

[12] J. Muramoto, T. Inmaru, Y. Nakata, T. Okada, and M. Maeda: Appl. Phys. A, 69, (1999) S239.

[13] J. Muramoto, Y. Nakata, T. Okada, and M. Maeda: Jpn J. Appl. Phys., 36, (1997) L563.

[14] D. B. Geohegan, A. A. Puretzky, G. Duscher, and S. J. Pennycook: Appl. Phys. Lett., 72, (1998) 2987.

[15] T. Makimura, T. Mizuta, and K. Murakami: Appl. Phys. A, 69, (1999) S213.

[16] J. Muramoto, I. Sakamoto, Y. Nakata, T. Okada, and M. Maeda: Appl. Phys. Lett., 75, (1999) 751.

[17] J. Muramoto, Y. Nakata, T. Okada, and M. Maeda: Appl. Surf. Sci., 127-129, (1998) 373.

[18] Y. Nakata, J. Muramoto, T. Okada, and M. Maeda: J. Appl. Phys., 91, (2002) 1640.

(Received: June 14, 2021, Accepted: December 24, 2021) 